\title{
The endothelin-1 G5665T polymorphism impacts transplant-free survival for single ventricle patients
}

\author{
Paul M. Kirshbom, MD, ${ }^{a}$ William T. Mahle, MD, ${ }^{\mathrm{b}}$ Ronald W. Joyner, MD, PhD, ${ }^{\mathrm{b}}$ Traci Leong, PhD, ${ }^{\mathrm{d}}$ Malania Wilson, MS, ${ }^{\mathrm{c}}$ \\ Brian E. Kogon, MD, ${ }^{a}$ Kirk R. Kanter, $M D^{a}{ }^{a}$ and Mark M. Bouzyk, $\mathrm{PhD}^{\mathrm{c}}$
}

Background: Early survival for children with single-ventricle congenital heart disease has improved, but late attrition remains a serious problem. The endothelin-1 G5665T single nucleotide polymorphism has been linked to increased vascular reactivity and hypertension. The goal of this study was to determine whether this single nucleotide polymorphism alters transplant-free survival for children with single-ventricle congenital heart disease.

Methods: DNA was isolated from 165 children with single-ventricle congenital heart disease born between January 1980 and December 2006. The endothelin-1 G5665T single nucleotide polymorphism genotype was determined by using real-time polymerase chain reaction. Kaplan-Meier survival curves were generated with a combined end point of death or transplantation. The Cox proportional hazard method was used to evaluate potential covariates.

Results: The endothelin-1 G5665T genotype was significantly associated with transplant-free survival for the group as a whole $(P=.002)$, with the greatest effect in children with hypoplastic left heart syndrome $(\mathrm{n}=64, P=.0002)$ as opposed to patients with other types of single-ventricle anatomy $(\mathrm{n}=101, P=.1)$. Cox proportional hazard modeling revealed 3 independent predictors of poor outcome: endothelin G5665T genotype (T/T genotype, $P=.001$ ), prematurity (gestational age $<37$ weeks, $P=$ .02 ), and era of surgical intervention (1990s vs other decades, $P=.02$ ).

Conclusions: Long-term survival of single-ventricle patients is dependent on many factors. These data suggest that genetic variability involving vascular resistance modifiers, such as endothelin-1, might play an important role, particularly in patients with hypoplastic left heart syndrome. Future studies should include evaluation of the

From the Division of Cardiothoracic Surgery, Department of Surgery, the Division of Cardiology, ${ }^{\mathrm{b}}$ Department of Pediatricsc; and the Departments of Human Genetics ${ }^{\mathrm{c}}$ and Biostatistics, ${ }^{\mathrm{d}}$ Emory University School of Medicine and Children's Healthcare of Atlanta, Atlanta, Ga.

Presented at the poster session of the 43rd Annual Meeting of The Society of Thoracic Surgeons, San Diego, Calif, January 29-31, 2007.

Received for publication May 21, 2007; revisions received Nov 14, 2007; accepted for publication Feb 25, 2008.

Address for reprints: Paul M. Kirshbom, MD, 1365 Clifton Rd, A2100, Atlanta, GA 30322 (E-mail: Paul.Kirshbom@ emoryhealthcare.org).

J Thorac Cardiovasc Surg 2008;136:117-22 0022-5223/\$34.00

Copyright $\odot 2008$ by The American Association for Thoracic Surgery

doi:10.1016/j.jtcvs.2008.02.040 relationship between endothelin genotype and plasma endothelin levels and possibly therapeutic trials of endothelin blockers in high-risk patients.

$\mathrm{T}$ The majority of children born with single-ventricle congenital heart disease (SV-CHD) undergo staged palliation culminating in the Fontan procedure during early childhood. Although the early survival of these children has steadily improved over the past decades, midterm to long-term attrition remains a significant concern. Failure of single-ventricle palliation is clearly multifactorial, as has been demonstrated by several large cross-sectional studies investigating the potential anatomic and treatment-related causes of Fontan failure. ${ }^{1,2}$ Although these studies have identified risk factors for early Fontan failure, such as younger age at surgical intervention, increased pulmonary artery pressures, heterotaxy syndrome, and longer cardiopulmonary bypass time, the results have been inconsistent and, to quote Gentles and colleagues, ${ }^{1}$ "A continuing late hazard phase is associated with few patientrelated variables and does not appear related to procedural variables." Although there is ample evidence that genetic factors can play a significant role in the development of cardiovascular disease and response to therapy in adults, ${ }^{3-6}$ there have been no studies reported regarding the effect of genetic factors on outcomes for single-ventricle patients. 


\section{Abbreviations and Acronyms \\ ET-1 = Endothelin-1 \\ HLHS = Hypoplastic left heart syndrome \\ SNP = Single nucleotide polymorphism \\ $\mathrm{SV}-\mathrm{CHD}=$ Single-ventricle congenital heart disease}

Considering the demonstrated sensitivity of single-ventricle patients to increased pulmonary vascular resistance and the potential for late single-ventricle dysfunction in the face of increased systemic vascular resistance, one area of particular interest for this patient population is vasomotor control. Endothelin-1 (ET-1) is one of the most potent endogenous vasoconstrictors known. A single nucleotide polymorphism (SNP) in the preproendothelin-1 gene involving a G-to-T replacement at nucleotide 5665 in exon 5 (rs5370; corresponding to a Lys/Asn change at codon 198) has been studied in several adult patient populations. The T allele (Asn198) is associated with increased vascular reactivity in human thoracic artery specimens in vitro, ${ }^{7}$ hypertension in obese populations, ${ }^{8}$ heart failure, ${ }^{9}$ and malignant arrhythmias in adults with structural cardiac disease. ${ }^{10}$

The goal of the present study was to determine whether the ET-1 G5665T polymorphism has any effect on midterm outcome for palliated single-ventricle patients. Our hypothesis was that children who are homozygous for the $\mathrm{T}$ allele would be at increased risk for midterm to late-term failure of singleventricle palliation compared with the more common $\mathrm{G}$ allele homozygotes and heterozygotes.

\section{Materials and Methods}

Emory University and Children's Healthcare of Atlanta Institutional Review Board approval was granted for this study. Informed consent was obtained from the patients' legal guardians, with patient assent obtained for children older than 6 years.

\section{Patient Population}

All patients born with SV-CHD presenting to Children's Healthcare of Atlanta, Egleston Hospital, between July 2004 and February 2007 were considered for enrollment in the study. The patient population included 2 discrete groups: (1) children who are currently undergoing staged palliation or who have completed staged palliation with a Fontan procedure and (2) patients with SV-CHD in whom single-ventricle palliation has previously failed and who underwent cardiac transplantation in the past. Patients at the first stage of palliation were enrolled before transfer out of the intensive care unit so as to eliminate first-stage operative mortality from this analysis of midterm to long-term outcomes. For patients who previously underwent transplantation, the date of transplantation was considered their end point. Those who underwent elective transplantation as infants without attempted staged palliation were excluded from the study because their genetic milieu could not be considered a contributor to the "failure" of single-ventricle palliation.
One hundred sixty-five patients were enrolled of 192 evaluated for the study. Patients were not enrolled for the following reasons: 11 parents either refused consent or could not be contacted, 8 were non-English speaking and adequate translation could not be arranged for informed consent, and 8 had elective neonatal heart transplantations without attempted staged palliation.

\section{DNA Isolation and Genotyping}

After obtaining informed consent, a 5-mL aliquot of whole blood was drawn during routine cardiac catheterization, during anesthesia induction for cardiac surgery, or from an arterial catheter in the postoperative period at least 24 hours after any exogenous blood exposure. Genomic DNA was isolated by using standard techniques (Puregene; Gentra Systems, Minneapolis, Minn). The ET-1 G5665T polymorphism was genotyped by using an Applied Biosystems Taqman 7000 analyzer (Foster City, Calif). The primers and probes used are shown in Table 1.

\section{Follow-up}

The parents/guardians of the patients have been contacted by telephone every 6 months for serial follow-up. Failure of single-ventricle palliation was defined as listing for cardiac transplantation or death. Patients who had cardiac transplantations before enrollment were assigned an end point date corresponding to the date of the transplantation and were not contacted beyond the date of enrollment.

\section{Statistical Analysis}

Data are presented as means \pm standard deviations. Categorical variables were compared by using $\chi^{2}$ analysis or the Fisher exact test, as appropriate. Continuous variables were compared by using the Kruskal-Wallis test.

Time to failure of single-ventricle palliation was calculated from the date of birth to the date of death or listing for cardiac transplantation. Transplant-free survival curves were calculated by using the Kaplan-Meier method and compared with the log rank test. The Cox proportional hazard model method was used to evaluate the joint effect of the studied polymorphism and potential covariates. A backward elimination multiple regression was used. At each step of model fitting, the Wald test was used to eliminate variables until all remaining variables had a $P$ value of less than or equal to .05 . All $P$ values were 2 -sided.

\section{Results}

Of the 165 patients enrolled, there were 109 male and 56 female patients. Birthweight was $3.2 \pm 0.6 \mathrm{~kg}$ (reliable data available for 161 patients). Cardiac anatomy at birth is summarized in Table 2. The group labeled "other singleventricle physiology" included a wide variety of anatomies, including double-outlet right ventricle variants with hypoplastic or atretic mitral valves, "upstairs/downstairs" ventricles with straddling atrioventricular valves, and others that did not fit the broader categories. A genetic syndrome was identified in $9(5 \%)$ of 165 patients, including 4 with Down syndrome, 2 with Turner's mosaic, and 1 each with DiGeorge syndrome, Klinefelter syndrome, and sickle cell anemia. Other patient demographics and characteristics are 
TABLE 1. Primers and probes used to genotype the endothelin-1 G5665T polymorphism

\begin{tabular}{ll}
\hline Primer/probe & \multicolumn{1}{c}{ Sequence } \\
\hline Forward & ACCATGAGAAACAGCGTCAAATCA \\
Reverse & GTGGGTCACATAACGCTCTCT \\
Vic & AGGCAAGCCCTCC \\
Fam & AAAGGCAATCCCTCC \\
\hline
\end{tabular}

Vic and Fam are Fluorescent Taqman reporter dyes (Applied Biosystems, Foster City, Calif).

summarized in Table 3, broken down by ET-1 G5665T genotype. For surviving patients who have not had a heart transplantation (those who were censored), the mean follow-up time was $4.3 \pm 4.1$ years (range, 125 days -22 years). The stage of palliation or end point achieved as of the last follow-up for the group as a whole is shown in Table 4.

Kaplan-Meier survival analysis demonstrates that the ET1 G5665T polymorphism is significantly associated with transplant-free survival $(P=.002$; Figure $1, A)$. Median actuarial survivals were 5,17 , and 25 years for the $\mathrm{T} / \mathrm{T}$ $(n=10), G / G(n=103)$, and $G / T(n=52)$ genotypes, respectively. Using a recessive gene model in which the $\mathrm{T} / \mathrm{T}$ group is compared against the other 2 groups $(\mathrm{G} / \mathrm{G}+\mathrm{G} / \mathrm{T})$ yields a stronger association with outcome $(P=.001$; Figure $1, B)$.

It is interesting to note that the observed effect of this polymorphism derives primarily from a transplant-free survival decrement in the subgroup of patients with hypoplastic left heart syndrome (HLHS) who have the T/T genotype (Figure 2). Kaplan-Meier analysis of patients with HLHS demonstrates a significant association between ET-1 G5665T genotype and survival ( $\mathrm{n}=64, P=.0002)$, as opposed to the single-ventricle patients with other cardiac anatomies, for whom there is not a significant association $(\mathrm{n}=101$, $P=.12)$. HLHS diagnosis in and of itself did not affect survival when compared against other diagnoses $(P=.76$, Figure 3$)$.

Cox proportional hazard analysis was performed, including the following variables: cardiac anatomy, single-ventricle morphology (right vs left ventricle), genetic syndromes,

\section{TABLE 2. Cardiac anatomy at birth}

\begin{tabular}{lrrrc}
\hline \multicolumn{1}{c}{ Cardiac anatomy } & G/G & G/T & T/T & No. (\%) \\
\hline Hypoplastic left heart syndrome & 38 & 20 & 6 & $64(39)$ \\
Hypoplastic RV with pulmonary & 9 & 6 & 2 & $35(21)$ \\
$\quad$ or tricuspid atresia & & & & \\
$\quad$ Double-inlet left ventricle & 24 & 11 & 0 & $17(10)$ \\
$\begin{array}{l}\text { Unbalanced atrioventricular } \\
\quad \text { canal defect }\end{array}$ & 6 & 3 & 0 & $9(5)$ \\
Heterotaxy syndrome & 4 & 5 & 1 & $10(6)$ \\
Other single-ventricle physiology & 22 & 7 & 1 & $30(18)$ \\
Total & 103 & 52 & 10 & $165(100)$ \\
\hline
\end{tabular}

$R V$, Right ventricle.
TABLE 3. Patient characteristics for endothelin-1 G5665T genotype groups

\begin{tabular}{lcccc}
\hline & G/G & $\mathbf{G / T}$ & $\mathbf{T / T}$ & $\boldsymbol{P}$ value \\
\hline Sex (\% male) & 63 & 75 & 50 & .18 \\
Race & & & & \\
$\quad$ White & 58 & 40 & 7 & \\
$\quad$ African-American & 29 & 6 & 1 & \\
$\quad$ Hispanic & 11 & 3 & 2 & \\
$\quad$ Other & 5 & 3 & 0 & .30 \\
Birthweight (kg) & $3.2 \pm 0.5$ & $3.2 \pm 0.6$ & $3.1 \pm 0.4$ & .97 \\
Premature (\%) & 18 & 10 & 0 & .45 \\
Ventricular & 37 & 44 & 20 & .33 \\
$\quad$ morphology (\% LV) & & & & \\
Genetic syndromes (\%) & 8 & 19 & 0 & .23 \\
Era (birth date) & & & & \\
$\quad$ 1980-1989 & 6 & 2 & 2 & \\
$\quad$ 1990-1999 & 20 & 11 & 2 & \\
$\quad$ 2000-2007 & 77 & 39 & 6 & .42 \\
\hline
\end{tabular}

Prematurity was defined as gestational age of less than 37 weeks. $L V$, Left ventricle.

birthweight, gestational age, era (grouped by birth date: 1980-1989, 1990-1999, and 2000-2006), ethnicity, sex, and ET-1 G5665T recessive model (T/T genotype vs "other"). This analysis produced a model with 3 significant independent predictors of transplant-free survival: ET-1 genotype (T/T vs "other"), prematurity, and era of birth $(P=$ $.001, .02$, and .02 , respectively). The higher-risk groups for these variables were T/T genotype, prematurity ( $<37$ weeks' gestational age), and birth date in the 1990s as opposed to the 1980s or 2000s. The distributions of genotypes across these variables are shown in Tables 2 and 3.

\section{Discussion}

Although the early survival for children with SV-CHD has improved dramatically over the past 2 decades, late attrition remains a significant concern. Gentles and colleagues ${ }^{1}$ reviewed the long-term results of 500 children who had Fontan

TABLE 4. Palliation stage completed or end point as of last follow-up

\begin{tabular}{lc}
\hline \multicolumn{1}{c}{ Palliation stage or end point } & No. (\%) \\
\hline $\begin{array}{l}\text { Stage } 1 \text { (eg, BT shunt, PA band, or Norwood } \\
\text { procedure) }\end{array}$ & $5(3)$ \\
Stage 2 (Glenn shunt or hemi-Fontan procedure) & $42(25)$ \\
Fontan procedure & $85(52)$ \\
Listed for transplantation or having undergone & $21(13)$ \\
$\quad$ transplantation & \\
Died & $12(7)$ \\
Total & $165(100)$ \\
\hline
\end{tabular}

$B T$, Blalock-Taussig; $P A$, pulmonary artery. 

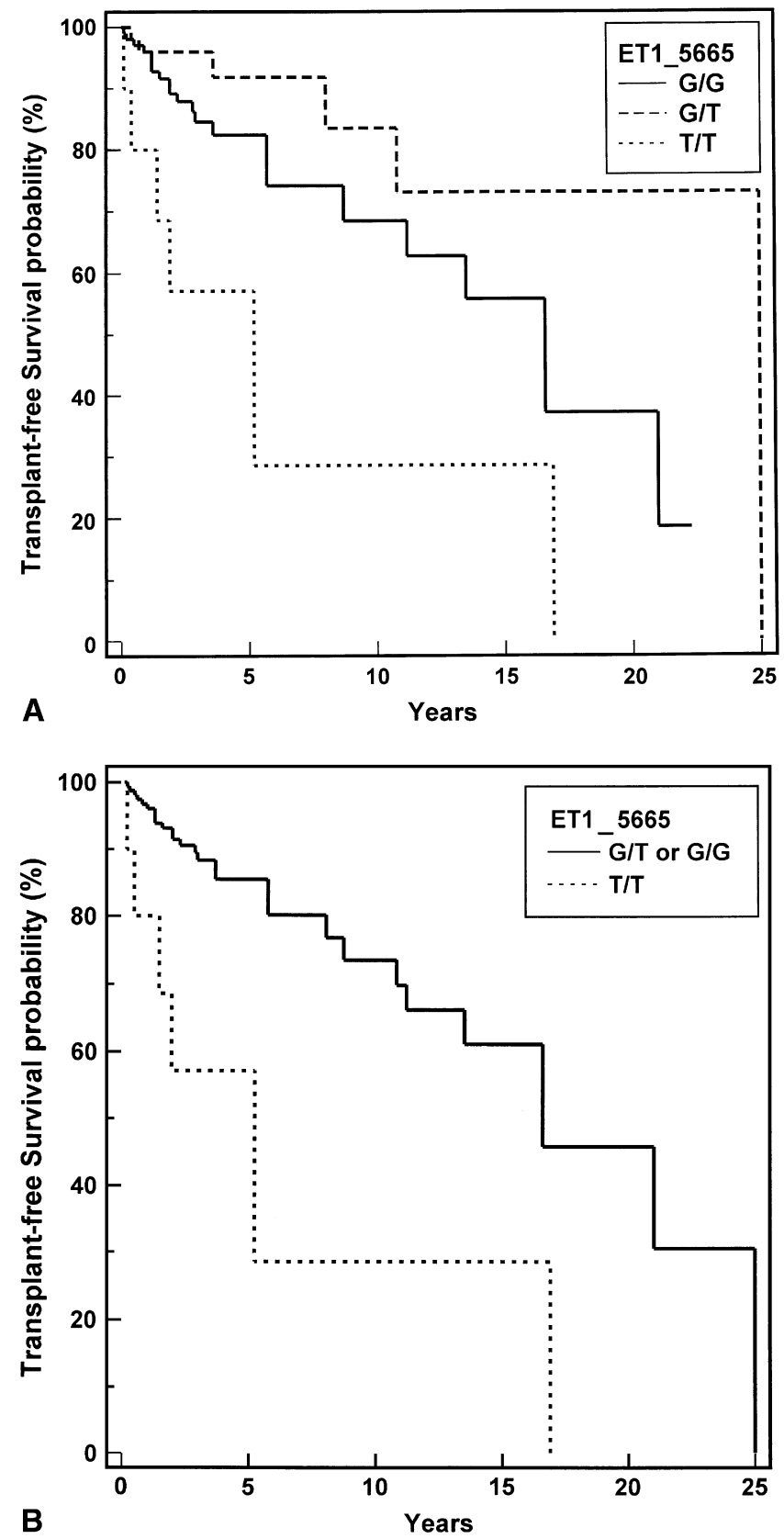

Figure 1. A, Transplant-free actuarial survival for all singleventricle patients stratified by endothelin-1 $\mathrm{G} 5665 \mathrm{~T}$ polymorphism genotype $(P=.002)$. $B$, Transplant-free actuarial survival for all single-ventricle patients using a recessive model for endothelin-1 G5665T polymorphism ( $T / T$ genotype vs $G / T$ and $G / G$ genotypes combined, $P=.001)$. ET1, Endothelin-1.

procedures between 1973 and 1991. Even excluding the early hazard phase of staged single-ventricle palliation by including only patients undergoing Fontan completion, the actuarial transplant-free survival at 10 years was approximately $71 \%$. Also, given the time period during which these patients underwent palliation, the longest follow-up groups were composed of relatively less complicated anatomic groups, such as patients with morphologic left ventricles and normally related great vessels. Although the outcomes for patients from more recent years might be better due to earlier staging and improvements in surgical and medical management, the increasing survival and progression through palliation of the more complicated single-ventricle variants, such as HLHS, might well offset any improvement in outcomes for the group as a whole. As such, investigations into the causes of late Fontan failure are vitally important.

Unfortunately, the results of the larger follow-up studies evaluating anatomic and perioperative risk factors for early and late Fontan failure have been inconsistent and incomplete. Although Gentles and colleagues ${ }^{1}$ found that cardiac anatomy was a significant predictor of late Fontan failure with complex variants, such as HLHS and heterotaxy syndrome, faring worse, Mitchell and associates ${ }^{2}$ found no association between anatomy and midterm outcome. In general, the causes of midterm to long-term Fontan failure have not been identified.

The ET-1 G5665T SNP has been linked to increased vascular reactivity and several cardiovascular disorders in adults. ${ }^{7-10}$ ET-1 is well known as a powerful vasoconstrictive agent that has long-lasting effects after nearly irreversible binding to its receptor. However, another mechanism of action has been identified at ET-1 concentrations that are physiologically relevant. At subthreshold concentrations (10-100 pmol/L), ET-1 has little or no direct effect on isolated vascular rings from rabbit aortas or human internal thoracic arteries. ${ }^{7,11}$ However, this concentration range of ET-1, which is comparable with circulating levels, does result in potentiation of catecholamine-induced vasoconstriction by low concentrations of norepinephrine or phenylephrine. Henrion and Laher ${ }^{11}$ demonstrated that this effect is endothelium independent and does not result from changes in calcium flux.

Iglarz and coworkers ${ }^{7}$ demonstrated that the ET-1 G5665T polymorphism alters the potentiation response of human internal thoracic artery rings to exogenous ET-1. The ET-1 G5665T genotype did not alter direct contraction to exogenous ET-1 at concentrations of greater than 100 $\mathrm{pmol} / \mathrm{L}$, but at subthreshold concentrations, arteries from patients with the $\mathrm{G} / \mathrm{T}$ and $\mathrm{T} / \mathrm{T}$ genotypes contracted more vigorously to low concentrations of phenylephrine than did those from patients with the $\mathrm{G} / \mathrm{G}$ genotype, suggesting that this polymorphism either alters vascular reactivity directly or is in linkage disequilibrium with another mutation that does so.

The results of the current study demonstrate that the ET-1 G5665T mutation is associated with a significant decrease in transplant-free survival for palliated single-ventricle patients $(P=.002)$, with the majority of the decrement occurring in patients with HLHS $(P=.0002)$. It is important to note that in this patient population HLHS was not a risk factor 

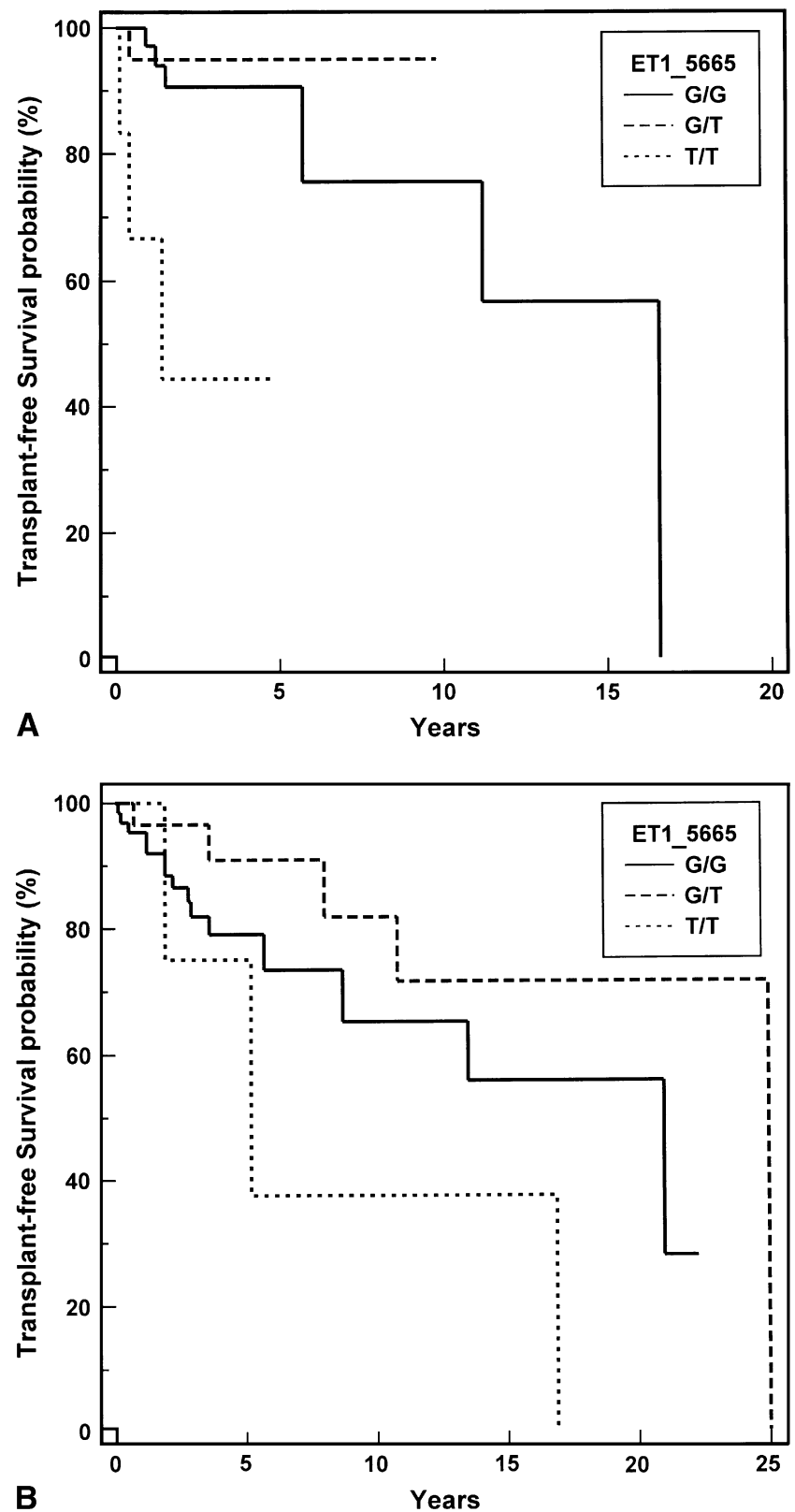

Figure 2. Transplant-free actuarial survival for patients with hypoplastic left heart syndrome $(A ; n=64, P=.0002)$ or other singleventricle anatomy ( $B ; n=101, P=.1$ ) stratified by endothelin-1 G5665T polymorphism genotype. ET1, Enothelin-1.

for decreased transplant-free survival in and of itself (Figure 3), but patients with HLHS seem to be preferentially vulnerable to the effects of this SNP. Whether this implies a higher sensitivity of patients with HLHS to increased vascular reactivity or another mechanism of action cannot be determined based on these data alone. Although the functional effect of this SNP has not been delineated, these data support the general supposition that afterload reduction might be use-

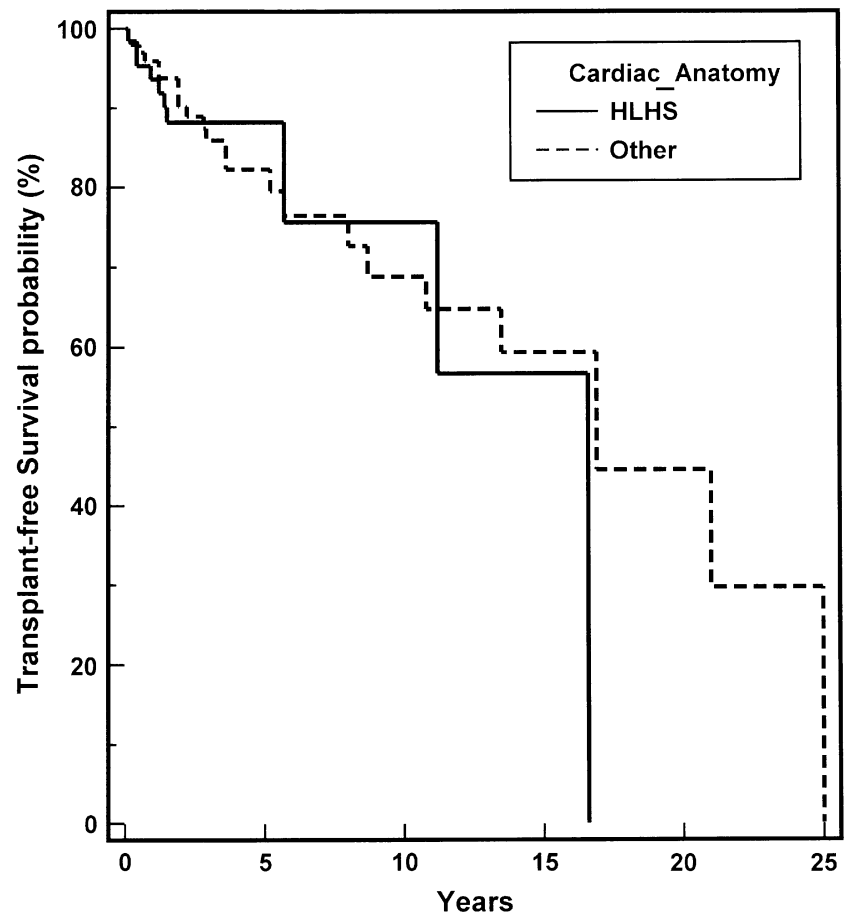

Figure 3. Transplant-free actuarial survival for single-ventricle patients stratified by cardiac diagnosis: hypoplastic left heart syndrome $(H L H S ; n=64)$ versus other single-ventricle anatomy $(n=$ 101). $P=.76$.

ful for long-term therapy of single-ventricle patients, particularly those with HLHS. It is also important to note that these data demonstrate an association between this polymorphism and outcome, but causality is only implied. These results would be entirely consistent with this SNP serving as a marker for another functionally significant mutation in linkage disequilibrium.

In our study the effects of the T allele did not appear to be additive because the $\mathrm{T} / \mathrm{T}$ group had significantly worse midterm transplant-free survival than the other groups, but the $\mathrm{G} /$ T heterozygotes were not significantly different from the $\mathrm{G} / \mathrm{G}$ homozygotes. This pattern is most consistent with a recessive gene model in which the poor T phenotype is only expressed in T homozygotes. Interestingly, this pattern was also demonstrated by Tiret and associates ${ }^{8}$ in their study of the effects of this same SNP on hypertension and obesity in adults. In that study the $\mathrm{G} / \mathrm{G}$ and $\mathrm{G} / \mathrm{T}$ subgroups had comparable blood pressures, whereas the $\mathrm{T} / \mathrm{T}$ subgroup had significantly higher systolic and diastolic blood pressures.

Our data must be considered within the limitations of the study design. Although many of the patients $(n=111)$ were enrolled prospectively as they presented to our institution for staged palliation, this study also included patients who were born and palliated in the past. For this older patient subgroup, the limitations of a retrospective study might apply. This 
older subgroup is also challenging in that it might represent opposing selection biases. On the one hand, there is a potential bias toward inclusion of longer-term survivors simply because those patients are still alive and available for enrollment. On the other hand, this group includes a large number of previously transplanted (or "failed") single-ventricle palliations $(n=24)$, and because the follow-up for patients undergoing transplantation is far more meticulous than for other patient groups, resulting in fewer patients lost to follow-up, there is a potential opposite bias toward better enrollment of early failures. Finally, although the subgroup analyses were interesting and suggestive, the number of patients within the anatomic subgroups was relatively small, and therefore further patient enrollment and follow-up will be necessary to increase statistical power before definitive statements can be made regarding the interaction between anatomy and genotype.

In conclusion, this study demonstrates that genetic factors can play a role in the long-term outcome of palliated singleventricle patients. Further studies are needed to confirm these findings in larger patient populations, as well as to evaluate the role of other candidate gene mutations. Given the relatively low incidence of the ET-1 $5665 \mathrm{~T} / \mathrm{T}$ genotype, a multicenter study would be required for any potential therapeutic trial of endothelin blockade in this apparently higher-risk group.

\section{References}

1. Gentles T, Mayer JJ, Gauvreau K, et al. Fontan operation in five hundred consecutive patients: factors influencing early and late outcome. J Thorac Cardiovasc Surg. 1997;114:376-91.

2. Mitchell M, Ittenbach R, Gaynor J, et al. Intermediate outcomes after the Fontan procedure in the current era. J Thorac Cardiovasc Surg. 2006; $131: 172-80$

3. Abraham M, Olson L, Joyner M, et al. Angiotensin-converting enzyme genotype modulates pulmonary function and exercise capacity in treated patients with congestive stable heart failure. Circulation. 2002;106: $1794-9$.

4. Andersson B, Sylven C. The DD genotype of the angiotensin-converting enzyme gene is associated with increased mortality in idiopathic heart failure. J Am Coll Cardiol. 1996;28:162-7.

5. Perez J, Rathz D, Petrashevskaya N, et al. $\beta 1$-adrenergic receptor polymorphisms confer differential function and predisposition to heart failure. Nat Med. 2003;9:1300-5.

6. Wagoner L, Craft L, Zengel P, et al. Polymorphisms of the beta1-adrenergic receptor predict exercise capacity in heart failure. Am Heart $J$. 2002;144:840-6.

7. Iglarz M, Benessiano J, Philip I, et al. Preproendothelin-1 gene polymorphism is related to a change in vascular reactivity in the human mammary artery in vitro. Hypertension. 2002;39:209-13.

8. Tiret L, Poirier O, Hallet V, et al. The Lys198Asn polymorphism in the endothelin-1 gene is associated with blood pressure in overweight people. Hypertension. 1999;33:1169-74.

9. Colombo M, Ciofini E, Paradossi U, et al. ET-1 Lys198Asn and ET (A) receptor $\mathrm{H} 323 \mathrm{H}$ polymorphisms in heart failure. A case-control study. Cardiology. 2006;105:246-52.

10. Kozak M, Izakovicova Holla L, Krivan L, et al. Endothelin-1 gene polymorphism in patients with malignant arrhythmias. J Cardiovasc Pharmacol. 2004;44(suppl):S92-5.

11. Henrion D, Laher I. Potentiation of norepinephrine-induced contractions by endothelin-1 in the rabbit aorta. Hypertension. 1993;22:78-83. 